\title{
Spectroscopic study of the inclusion compound of $\beta$-cyclodextrin and Tris(dibenzoylmethane)europium(III) dihydrate
}

\author{
H.F. Brito ${ }^{\text {a, C.A.A. Carvalho }}{ }^{\text {b,* }}$, O.L. Malta ${ }^{\text {c }}$, J.J. Passos ${ }^{\text {b }}$, J.F.S. Menezes ${ }^{\text {a }}$, \\ R.D. Sinisterra ${ }^{\mathrm{d}}$ \\ a Instituto de Química da Universidade de São Paulo, 05599-970 Sao Paulo, Brazil \\ ${ }^{\mathrm{b}}$ Departamento de Química da Universidade Federal de Ouro Preto, Departamento de Química, \\ Universidade Federal de Ouro Preto, Campus Morro de Cruzeiro, 35400-000 Ouro Preto MG, Brazil \\ c Departamento de Química Fundamental da Universidade Federal de Pernambuco, Recife, Brazil \\ d Departamento de Química da Universidade Federal de Minas Gerais, Belo Horizonte, Brazil
}

Received 15 January 1999; accepted 27 January 1999

\begin{abstract}
Photoluminescent properties of the inclusion compound of $\beta$-cyclodextrin and tris(dibenzoylmethane)europium(III) dihydrate, in the solid state, are reported. This compound and the precursor salt, $\mathrm{Eu}(\mathrm{DBM})_{3} \cdot 2 \mathrm{H}_{2} \mathrm{O}(\mathrm{DBM}=$ dibenzoylmethane), were characterized by IR spectroscopy, X-ray powder diffraction and differential scanning calorimetry. The emission data indicate that the host-guest interaction (at $77 \mathrm{~K}$ ) causes an intensification of some bands corresponding to the ${ }^{5} \mathrm{D}_{0} \rightarrow{ }^{7} \mathrm{~F}_{J}(J=0-4)$ transitions associated with one of the site symmetries existing in the guest compound. Based on the luminescence spectrum of the supramolecular compound the $\Omega_{\lambda}$ experimental intensity parameters $\left(\lambda=2\right.$ and 4) were calculated for the electronic transitions ${ }^{5} \mathrm{D}_{0} \rightarrow{ }^{7} \mathrm{~F}_{2,4}$. The value of the $\Omega_{2}$ intensity parameter for this new compound is smaller than for the guest, suggesting an effective interaction between the $\beta$-cyclodextrin and the closer chemical environment of the $\mathrm{Eu}^{3+}$ ion. On the other hand, the values of the $\Omega_{4}$ parameter for the inclusion compound are twice larger than the one of the guest, suggesting that the steric effect is more significant in the supramolecular species. Comparison between the luminescence lifetimes of the guest and inclusion compound indicates that the $\beta$-cyclodextrin contributes to increase the lifetime of the ${ }^{5} \mathrm{D}_{0}$ emitting level. The time-resolved spectra of the inclusion compound corroborate the presence of more than one site symmetry occupied by the rare earth ion. (C) 1999 Elsevier Science B.V. All rights reserved.
\end{abstract}

Keywords: Trivalent europium; $\beta$-cyclodextrin; Luminescence spectroscopy

\section{Introduction}

An important development in the field of the supramolecular chemistry is the study of com-

* Corresponding author. Fax: + 55-31-5591707. plexes of the type host-guest. Macrocyclic com-

E-mail address: caac@iceb.ufop.br (C.A.A. Carvalho) pounds are used to encapsulate chemical species 
in cavities with appropriate sizes. The supramolecular systems, in relation to the individual species, can be characterized by non covalent bonds and exhibit new physical and chemical properties [1,2].

The cyclodextrins have shown to be promising hosts in inclusion chemistry, presenting properties based on its steric conformation with conical external structures including a relatively large internal cavity. The external surface of these molecules is hydrophilic, while the internal cavity presents apolar character [1]. Cyclodextrins (CD) are cyclic oligosaccharides composed of six, seven and eight glucose units, known as $\alpha, \beta, \gamma-\mathrm{CD}$, respectively. They are composed of D-glucopyranose units linked by an $\alpha(1-4)$ glycoside linkage. In accord with its dimensions and hydrophilic-lipophilic properties several classes of chemical species can be included totally or partially in the cavity of the cyclodextrins. In the case of formation of the inclusion complex the physical and chemical properties of the guest can be modified as a consequence of the type of interaction host:guest. Consequently the cyclodextrins can be used in many fields as pharmaceutical preparations, cosmetic and food industries, separations and these host molecules can be utilized as model systems to study weak interactions [1-4].

The trivalent rare earth ions have been extensively studied in the last four decades by absorption and luminescence spectroscopy $[5,6]$. The electronic spectra of rare earth ions present sharp lines, a feature similar to the atomic or free gaseous ion spectra. This property is a direct consequence of the fact that the $4 \mathrm{f}$ electrons are screened from the chemical environment by the outer core of $5 \mathrm{~s}$ and $5 \mathrm{p}$ shells and $\mathrm{f}-\mathrm{f}$ transitions are forbidden by parity. This small perturbation in the free ion levels by the ligand field has been used to investigate the chemical environment around the central metal ion that can act as a local probe $[6,7]$.

Among the trivalent rare earth ions, europium and terbium present the most intense luminescence. The $\mathrm{Eu}^{3+}$ ion is commonly used as a probe due to its principal emitting level, ${ }^{5} \mathrm{D}_{0}$ and ground state, ${ }^{7} \mathrm{~F}_{0}$, which are non-degenerate, facilitating the interpretation of the spectral data as compared to the $\mathrm{Tb}^{3+}$ ion [5].
The goal of this work is to investigate the influence of the host-guest interaction between $\beta$-cyclodextrin and $\mathrm{Eu}(\mathrm{DBM})_{3} \cdot 2 \mathrm{H}_{2} \mathrm{O}$, in the solid state, on the optical properties of the inclusion compound (luminescence spectrum, emission lifetimes and energy transfer processes between host and guest). The data indicate that the guest can be accommodated in the host in at least two different ways.

\section{Experimental}

$\beta$-Cyclodextrin hydrate was supplied by Aldrich and used without further purification. The $\mathrm{Eu}(-$ $\mathrm{DBM})_{3} \cdot 2 \mathrm{H}_{2} \mathrm{O}$ compound was prepared by the same method reported in the literature [8].

The inclusion compound was prepared by the co-precipitation method, where a $\beta$-cyclodextrin aqueous solution was mixed dropwise with an ethanolic solution of rare earth salt under constant stirring. The reaction was kept at ca. 40$50^{\circ} \mathrm{C}$ for $24 \mathrm{~h}$, the final volume being reduced to ca. $10 \mathrm{ml}$. The white solid produced was collected by filtration and washed with water and dried in vacuum at room temperature.

The physical mixture of $\beta$-cyclodextrin and $\mathrm{Eu}(\mathrm{DBM})_{3} \cdot 2 \mathrm{H}_{2} \mathrm{O}$ in the molar ratio $1: 1$ was also prepared by gentle grinding of the components until a homogeneous powder was obtained.

X-ray powder patterns were obtained using a RIGAKU diffractometer filtered with a LiF monochromator and employing the $\mathrm{CuK} \alpha$ radiation as a source $(15 \mathrm{kV}, 15 \mathrm{~mA})$. The DSC curves were obtained on TA Instruments DSC-2010 with a heating rate of $10^{\circ} \mathrm{C} \mathrm{min}^{-1}$. under a nitrogen atmosphere. The IR spectra were registered in a MATTSON FTIR-3500, in $\mathrm{KBr}$ pellets, in the range of $4000-500 \mathrm{~cm}^{-1}$.

The excitation and emission spectra were recorded in a spectrofluorometer SPEX-Fluorolog 2 with double grating $0.22 \mathrm{~m}$ SPEX 1680 monochromators, a $450 \mathrm{~W}$ xenon lamp as excitation source and this apparatus was fully controlled by a DM3000F software spectroscopic computer. The lifetime curves and time-resolved espectra were obtained at 298 and $77 \mathrm{~K}$ using the Phosphorimeter Spex model 1934D. 


\section{Results and discussion}

DCS curves of $\mathrm{Eu}(\mathrm{DBM})_{3} \cdot 2 \mathrm{H}_{2} \mathrm{O}, \beta$-cyclodextrin, inclusion compound and physical mixture are shown in Fig. 1. At the same time DSC curves of the physical mixture and inclusion compound were compared and it was verified that the former curve showed two endothermic peaks, around 223 and $234^{\circ} \mathrm{C}$, non present in the curve of the isolated solid. Both peaks are observed in the curves of the host and guest, respectively, suggesting a thermal behavior for the physical mixture that is

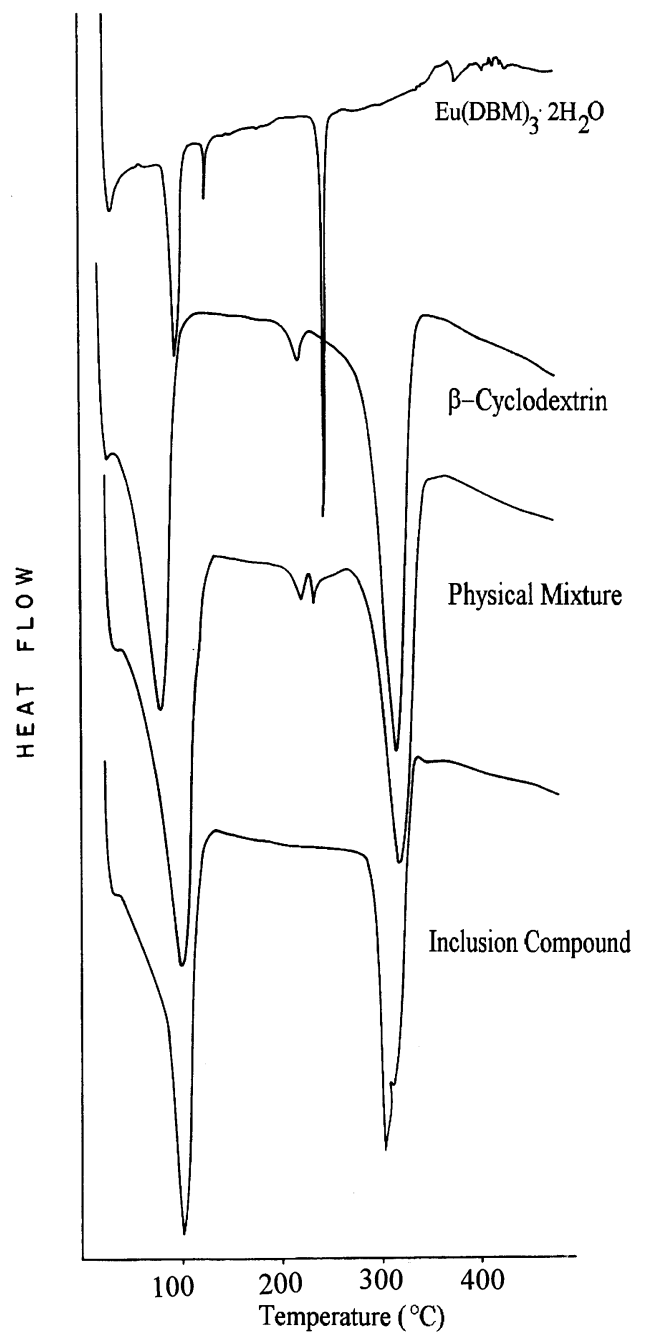

Fig. 1. DSC curves of the precursor salt, $\beta$-cyclodextrin, physical mixture and inclusion compound.

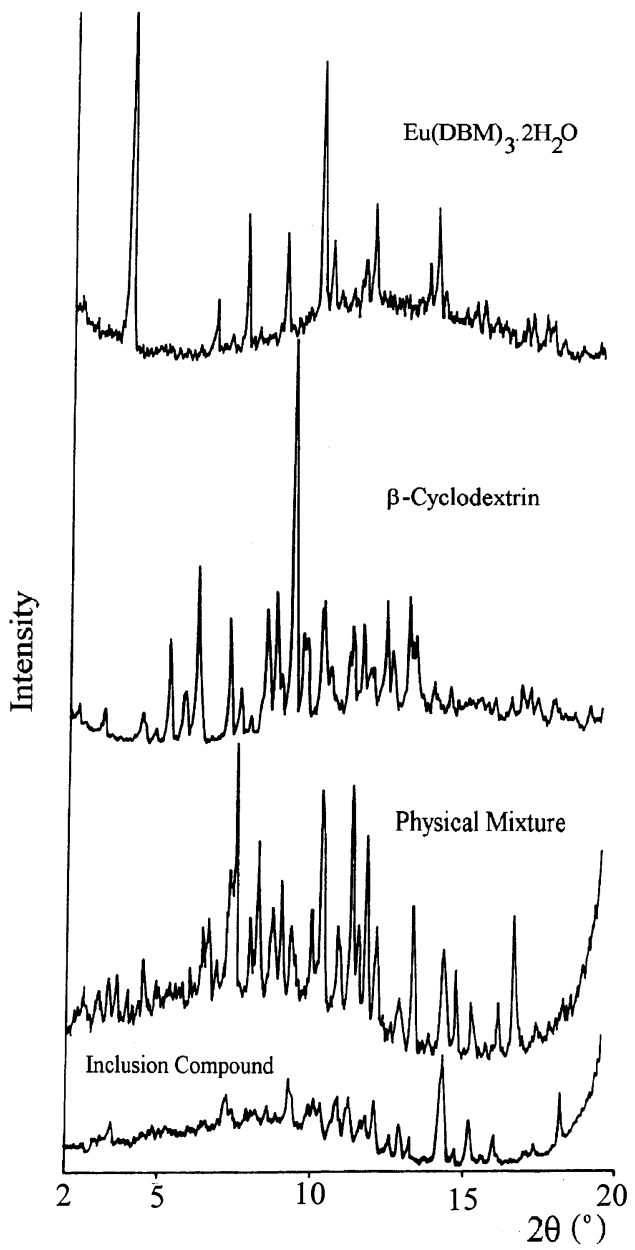

Fig. 2. XRD patterns of the precursor salt, $\beta$-cyclodextrin, physical mixture and inclusion compound.

approximately a superposition of the curves registered for the isolated components.

The endothermic peak at $223^{\circ} \mathrm{C}$ present in the DSC curve of the host and physical mixture can be assigned to a reversible transformation within the $\beta$-cyclodextrin molecule [9] that, by the interaction host-guest, vanish in the curve of the inclusion compound. On the other hand, the endothermic peak at $234^{\circ} \mathrm{C}$, present in the DSC curve of the guest and physical mixture, is due to the melting of the material. The thermal analysis data indicate the formation of the inclusion compound. 
The X-ray powder patterns of the inclusion compound, $\beta$-cyclodextrin, rare earth salt and physical mixture are presented in Fig. 2. The XRD results suggest a less crystalline character for the inclusion product, in contrast with the physical mixture, rare earth salt, or $\beta$-cyclodextrin. This lowering of the crystalline character is possibly a consequence of the loss of water molecules from the host. In the diffractogram of the supramolecular species the peaks at 5.4, 6.3, $7.4,9.0,9.5$ and $10.4^{\circ}$ in the $\beta$-cyclodextrin diffractogram are absent and those at 4.0, 6.8, 7.9, 9.2 and $10.4^{\circ}$ are present in the diffractogram of $\mathrm{Eu}(\mathrm{DBM})_{3} \cdot 2 \mathrm{H}_{2} \mathrm{O}$.

On the other hand, the diffractogram of the physical mixture is approximately the superposition of the diffractograms of the individual components. These evidences point to the formation of the supramolecular compound by the interaction of the rare earth salt with the $\beta$-cyclodextrin cavity.

The IR spectra for the inclusion compound, $\beta$-cyclodextrin, rare earth salt and physical mixture are presented in Fig. 3. Although the IR technique is not suitable for detection of formation of the inclusion compound [10], in this case it was possible to use it because the aromatic ring vibrational modes of the complex undergo a drastic modification in their profiles as compared to the guest spectrum, suggesting that the host-guest interaction occurs through the aromatic moiety of the ligand (DBM). In accordance with the thermal analysis and X-ray results, the physical mixture spectrum can be considered as a superposition of the individual component spectra. These evidences reinforce the conclusions from the DSC curves and X-ray diffraction measurements.

Fig. 4 shows the excitation spectra monitoring the ${ }^{5} \mathrm{D}_{0} \rightarrow{ }^{7} \mathrm{~F}_{2}$ hypersensitive transition at 611.2 $\mathrm{nm}$, except for the inclusion compound at low temperature $\left(\lambda_{\mathrm{em}}=623 \mathrm{~nm}\right)$. Comparison between the excitation spectra (Fig. 4) of the precursor salt and inclusion compound shows that there are significant differences. The spectrum of the inclusion compound shows a shift of the ligand states towards the higher energy region due to the interaction of the precursor salt with the $\beta$-cyclodextrin cavity.
Fig. 5 presents the emission spectra of the inclusion compound in the solid state at room and liquid nitrogen temperatures. For the sake of comparison with the corresponding spectrum at $298 \mathrm{~K}$, it is also shown the spectrum of the precursor salt at $77 \mathrm{~K}$. These results indicate that no significant structural changes occur in the $\mathrm{Eu}(-$ $\mathrm{DBM})_{3} \cdot 2 \mathrm{H}_{2} \mathrm{O}$ compound as the temperature is lowered. All the spectra present sharp lines characteristic of the energy level structure of $\mathrm{Eu}^{3+}$ ion, ${ }^{5} \mathrm{D}_{0} \rightarrow{ }^{7} \mathrm{~F}_{J}(J=0-4)$, with direct excitation of a $4 \mathrm{f}$ level, ${ }^{5} \mathrm{~L}_{6}$, around $394 \mathrm{~nm}$.

When we compare the emission spectra of the precursor salt and inclusion compound, for the ${ }^{5} \mathrm{D}_{0} \rightarrow{ }^{7} \mathrm{~F}_{J}$ transitions, it is observed a significant change in the profile and splitting of these bands, which corroborates the conclusions obtained by the characterization techniques indicating the formation of the inclusion complex.

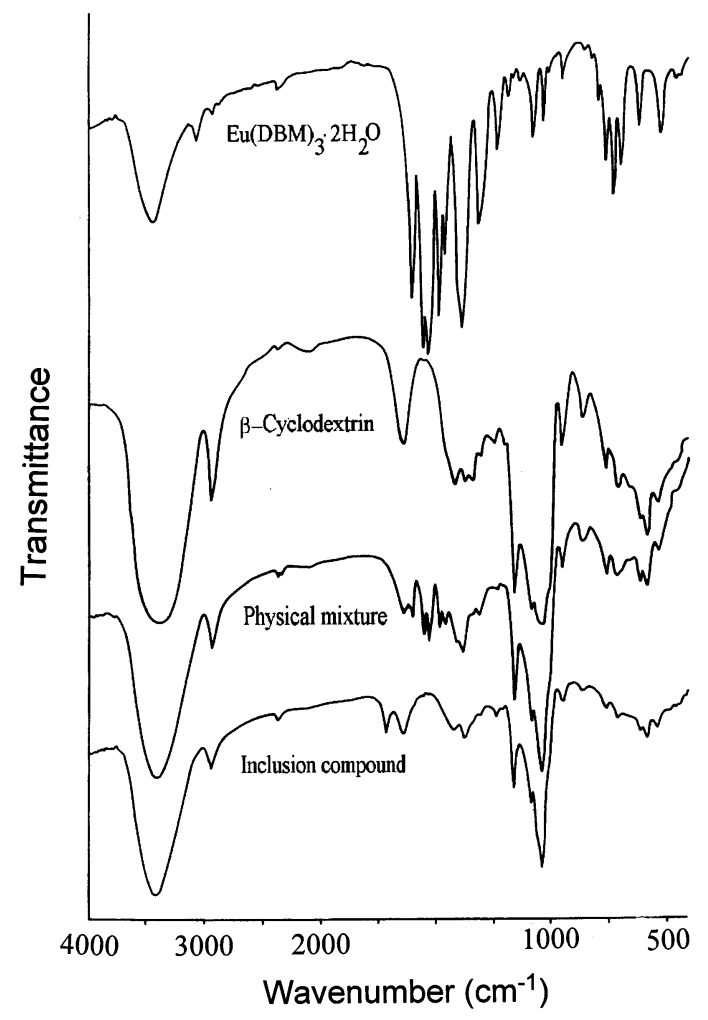

Fig. 3. Infrared spectra of the precursor salt, $\beta$-cyclodextrin, physical mixture and inclusion compound, in the solid state. 


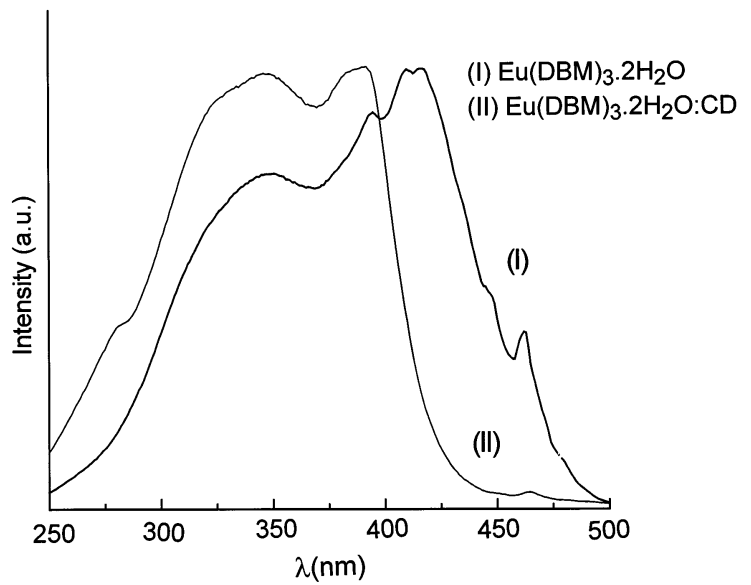

Fig. 4. Excitation spectra of the inclusion compound and precursor salt at room temperature. The emission was monitored at $611 \mathrm{~nm}$.

Inspection of the spectra in Fig. 5, in the region of the ${ }^{5} \mathrm{D}_{0} \rightarrow{ }^{7} \mathrm{~F}_{0}$ transition, shows the following features: (i) the peak at $576.2 \mathrm{~nm}$ presents a much higher intensity for the inclusion compound, at 77 $\mathrm{K}$, when compared with very weak intensity of the peak for the precursor salt; and (ii) in the case of the peak at $579 \mathrm{~nm}$ it is observed an inversion of relative intensities, with a decreasing factor of approximately 10 for the inclusion compound.

The same effect occurs for the ${ }^{5} \mathrm{D}_{0} \rightarrow{ }^{7} \mathrm{~F}_{1}$ transitions as noted by the increase of the area under the peaks in the range from 582 to $600 \mathrm{~nm}$ for the inclusion compound at $77 \mathrm{~K}$. However, some peaks in this region are quenched, when compared with the precursor salt.

In the case of ${ }^{5} \mathrm{D}_{0} \rightarrow{ }^{7} \mathrm{~F}_{2}$ transition it is observed that the peak at $612 \mathrm{~nm}$, for the salt, presents a decrease in intensity for the inclusion compound at $77 \mathrm{~K}$, whereas the peaks around 617 and 623 $\mathrm{nm}$ become more intense in the inclusion compound. It is observed in the ${ }^{5} \mathrm{D}_{0} \rightarrow{ }^{7} \mathrm{~F}_{4}$ transition that there is also an inversion in the intensity of some peaks at $77 \mathrm{~K}$.

The number of components for ${ }^{5} \mathrm{D}_{0} \rightarrow{ }^{7} \mathrm{~F}_{J}$ transitions $(J=0-2)$ in these spectra indicate that there is more then one site symmetry occupied by the $\mathrm{Eu}^{3+}$ in both inclusion compound and precursor salt. This is more precisely indicated in Table 1.
From Fig. 5 it may be seen that there is a similarity between the spectra of the inclusion compound $(298 \mathrm{~K})$ and the precursor salt $(77 \mathrm{~K})$ which is not verified for the inclusion compound at low temperature, where it occurs the inversion of the relative intensities. The intensification of some peaks in the ${ }^{5} \mathrm{D}_{0} \rightarrow{ }^{7} \mathrm{~F}_{J}$ transitions for the inclusion compound at $77 \mathrm{~K}$ is due probably to the steric effects and confinement of the guest into the cavity of the $\beta$-cyclodextrin that can behave as a second coordination sphere.

Based on the above results we can suggest that the $\mathrm{Eu}^{3+}$ ions are found in an environment of very low symmetry, as indicated by the number of lines for each electronic transition. A relevant feature in these spectra is that the cyclodextrin is acting as a selective medium changing drastically

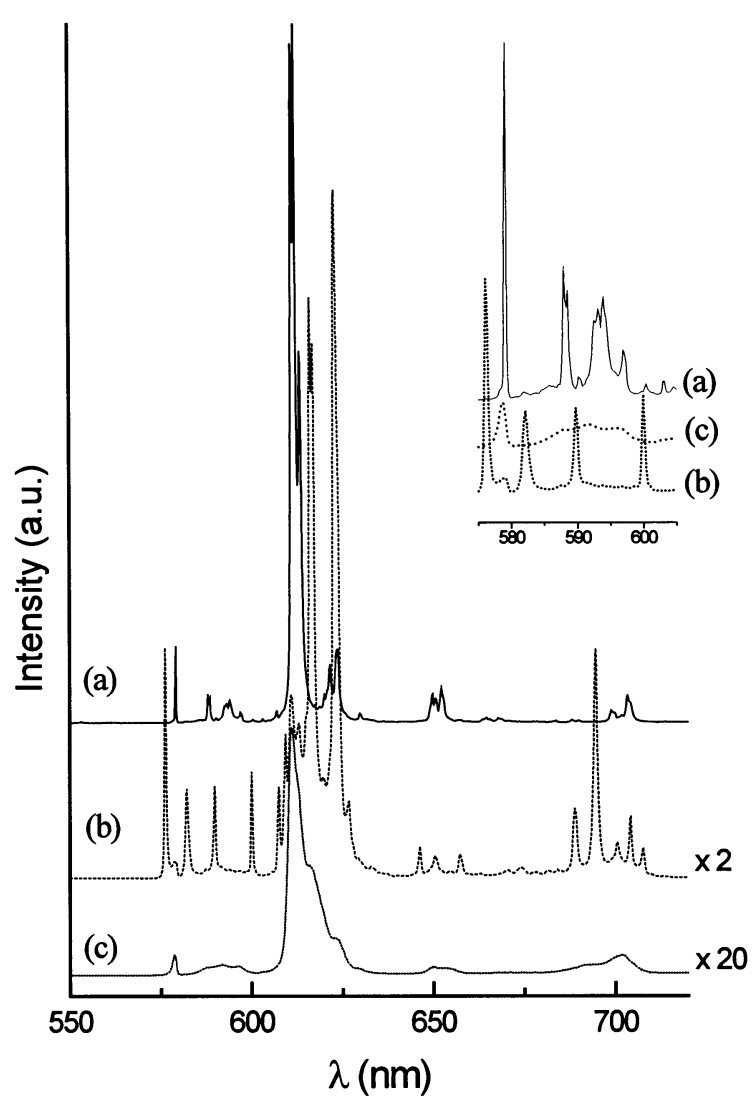

Fig. 5. Emission spectra of the inclusion compound: (a) at 298; (b) 77; and (c) precursor salt (77 K), under excitation at 394 $\mathrm{nm}$; the inset shows the ${ }^{5} \mathrm{D}_{0} \rightarrow{ }^{7} \mathrm{~F}_{0-1}$ spectral region amplified. 
Table 1

Energies of the ${ }^{5} \mathrm{D}_{0} \rightarrow{ }^{7} \mathrm{~F}_{0-4}$ transitions (in $\mathrm{cm}^{-1}$ ) observed in the emission spectra of the precursor salt and inclusion compound at $77 \mathrm{~K}$

\begin{tabular}{|c|c|c|}
\hline Transitions & Precursor salt & Inclusion compound \\
\hline${ }^{5} \mathrm{D}_{0} \rightarrow{ }^{7} \mathrm{~F}_{0}$ & $\begin{array}{l}17349 \\
17295^{*} \\
17265^{*}\end{array}$ & $\begin{array}{l}17355^{*} \\
17283 \\
17265\end{array}$ \\
\hline${ }^{5} \mathrm{D}_{0} \rightarrow{ }^{7} \mathrm{~F}_{1}$ & $\begin{array}{l}17182 \\
17006 \\
16989 \\
16943 \\
16874 \\
16857 \\
16835 \\
16784 \\
16750 \\
16655 \\
16540\end{array}$ & $\begin{array}{l}17176^{*} \\
17024 \\
16995^{*} \\
16903 \\
16841 \\
16756 \\
16705 \\
16666^{*} \\
16600\end{array}$ \\
\hline${ }^{5} \mathrm{D}_{0} \rightarrow{ }^{7} \mathrm{~F}_{2}$ & $\begin{array}{l}16474 \\
16420 \\
16361 \\
16339 \\
16302 \\
16197 \\
16160 \\
16129 \\
16108 \\
16087 \\
16036 \\
15984 \\
15878 \\
15827\end{array}$ & $\begin{array}{l}16474 \\
16410 \\
16367 \\
16339 \\
16313 \\
16260 \\
16228 \\
16207 \\
16144 \\
16134 \\
16051 \\
15959 \\
15893 \\
15802\end{array}$ \\
\hline${ }^{5} \mathrm{D}_{0} \rightarrow{ }^{7} \mathrm{~F}_{3}$ & $\begin{array}{l}15332 \\
15365 \\
15389\end{array}$ & $\begin{array}{l}15475 \\
15375 \\
15216\end{array}$ \\
\hline${ }^{5} \mathrm{D}_{0} \rightarrow{ }^{7} \mathrm{~F}_{4}$ & $\begin{array}{l}14306 \\
14289 \\
14249 \\
14216\end{array}$ & $\begin{array}{l}14518 \\
14397 \\
14278 \\
14201 \\
14132\end{array}$ \\
\hline
\end{tabular}

* From amplified spectral region.

the relative intensities of the Stark components of the ${ }^{5} \mathrm{D}_{0} \rightarrow{ }^{7} \mathrm{~F}_{J}$ transitions.

The time-resolved spectra were obtained for the inclusion compound at $77 \mathrm{~K}$ (Fig. 6) with the objective of verifying the possibility of occurrence of transitions arising from excited levels above the ${ }^{5} \mathrm{D}_{0}$. It was noticed that the band at $582.2 \mathrm{~nm}$ could be assigned to the ${ }^{5} \mathrm{D}_{1} \rightarrow{ }^{7} \mathrm{~F}_{3}$ transition [11]. The ${ }^{5} \mathrm{D}_{1}$ emitting level present a much shorter lifetime when compared with the ${ }^{5} \mathrm{D}_{0}$ level. These results were corroborated by the lifetimes data where all the values are characteristic of the ${ }^{5} \mathrm{D}_{0}$ level. This is shown in Table 2. The intensities of the bands corresponding to the transitions ${ }^{5} \mathrm{D}_{0} \rightarrow$ ${ }^{7} \mathrm{~F}_{0}(\sim 579 \mathrm{~nm})$ and ${ }^{5} \mathrm{D}_{0} \rightarrow{ }^{7} \mathrm{~F}_{2}(\sim 612 \mathrm{~nm})$, indicated by arrows, are decreased when the spectra are recorded with different delay times (Fig. 6). These changes in the intensities suggest that those are caused by simultaneous excitation of $\mathrm{Eu}^{3+}$ ions in more than one site symmetry.

The time resolved emission spectra for the precursor salt were recorded at $77 \mathrm{~K}$ with interval delays of $0.1-1.0 \mathrm{~ms}$.

The lifetimes of the inclusion compound at 77 $\mathrm{K}$ showed three different values $(\tau=\sim 0.64, \sim$ 0.42 and $\sim 0.22 \mathrm{~ms}$ ) for the ${ }^{5} \mathrm{D}_{0}$ emitting level (Table 2), while the precursor salt present only one lifetime ( $\tau=\sim 0.23 \mathrm{~ms}$ ). This also corroborates the fact that the precursor salt is confined in more than one site symmetry in the inclusion compound. However, it is also plausible that the differences in the lifetimes observed in the inclusion compound have a contribution from an energy transfer pathway involving the $\mathrm{Eu}^{3+}$ ion and a localized electronic state of the $\beta$-cyclodextrin.

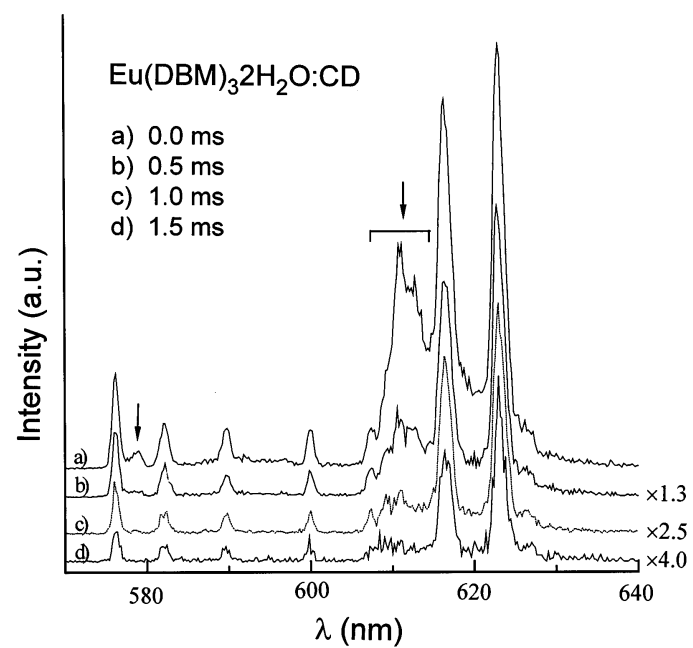

Fig. 6. Time-resolved emission spectra of the ${ }^{5} \mathrm{D}_{0} \rightarrow{ }^{7} \mathrm{~F}_{J}(J=0$, 1 and 2) transitions for the inclusion compound monitored under $394 \mathrm{~nm}$ excitation at $77 \mathrm{~K}$. The time delays following excitation are indicated for each spectrum. 
Table 2

Experimental intensity parameters $\Omega_{2}, \Omega_{4}$ and lifetimes $(\tau)$ for the $\mathrm{Eu}(\mathrm{DBM})_{3} \cdot 2 \mathrm{H}_{2} \mathrm{O}$ and $\mathrm{Eu}(\mathrm{DBM})_{3} \cdot 2 \mathrm{H}_{2} \mathrm{O}: \mathrm{CD}$ compounds, where $\mathrm{CD}=\beta$-cyclodextrin, at $77 \mathrm{~K}$

\begin{tabular}{lllr}
\hline Compounds & $\Omega_{2}\left(10^{-20} \mathrm{~cm}^{2}\right)$ & $\Omega 4\left(10^{-20} \mathrm{~cm}^{2}\right)$ & $\tau(\mathrm{ms})$ \\
\hline $\mathrm{Eu}(\mathrm{DBM})_{3} \cdot 2 \mathrm{H}_{2} \mathrm{O}$ & 32.22 & 3.63 & 0.23 \\
$\mathrm{Eu}(\mathrm{DBM})_{3} \cdot 2 \mathrm{H}_{2} \mathrm{O}: \mathrm{CD}$ & 21.7 & 8.7 & $\sim 0.64, \sim 0.42, \sim 0.22$ \\
\hline
\end{tabular}

The emission intensity, $I$, of a given transition is proportional to the surface under the emission curve: $I=\hbar \omega A N$, where $\hbar \omega$ is the transition energy and $N$ is the population of the emitting level, ${ }^{5} \mathrm{D}_{0}$. From the luminescence spectra at $298 \mathrm{~K}$ we have calculated the experimental intensity parameters, $\Omega_{2}$ and $\Omega_{4}$, for the inclusion and guest compounds, by using the ${ }^{5} \mathrm{D}_{0} \rightarrow{ }^{7} \mathrm{~F}_{2}$ and ${ }^{5} \mathrm{D}_{0} \rightarrow{ }^{7} \mathrm{~F}_{4}$ transitions, respectively. The Einstein coefficient of spontaneous emission, $A$, is given by $[12,13]$.

$A=\frac{4 e^{2} \omega^{3}}{3 \hbar c^{3}}(2 J+1)^{-1} \chi \sum_{\lambda} \Omega_{\lambda}\left\langle{ }^{7} \mathrm{~F}_{J}\left\|\mathrm{U}^{(\lambda)}\right\|{ }^{5} \mathrm{D}_{0}\right\rangle^{2}$

where $\chi=n_{0}\left(n_{0}^{2}+2\right)^{2} / 9$ is a Lorentz local field correction, $n_{0}$ being the index of refraction of the medium. The magnetic dipole allowed ${ }^{5} \mathrm{D}_{0} \rightarrow{ }^{7} \mathrm{~F}_{1}$ transition was taken as the reference. The reduced matrix elements in Eq. (1) were taken from Carnall et al. [14] and an average index of refraction equal to 1.5 was assumed.

The value of the $\Omega_{2}$ intensity parameter for the inclusion compound is smaller than that obtained for the guest. For both compounds the $\Omega_{2}$ values are very high [15], reflecting the hypersensitive character of the ${ }^{5} \mathrm{D}_{0} \rightarrow{ }^{7} \mathrm{~F}_{2}$ transition and indicating that europium (III) ions are in a highly polarizable chemical environment. On the other hand, the value of the $\Omega_{4}$ parameter for the inclusion compound is twice larger than the one for the guest. These results indicate that the steric effect is more significant in the supramolecular species, as expected and also corroborates the influence of the cyclodextrin, as a second coordination sphere, on the $\mathrm{Eu}^{3+}$ ion.

\section{Concluding remarks}

The characterization techniques used in the present work have pointed to the formation of the inclusion compound involving the $\beta$-cyclodextrin and the $\mathrm{Eu}(\mathrm{DBM})_{3} \cdot 2 \mathrm{H}_{2} \mathrm{O}$ compound. The ${ }^{5} \mathrm{D}_{0} \rightarrow$ ${ }^{7} \mathrm{~F}_{0}$ transition for the inclusion compound indicated more than one site symmetry occupied by the rare earth ion. The lifetime results indicated that the $\beta$-cyclodextrin recognized the three distinct lifetimes due probably to the contribution of different site symmetries being occupied by the rare earth ion and also due to a possible energy transfer pathway involving the cyclodextrin and the $\mathrm{Eu}^{3+}$ ion. These observations show that the $\beta$-cyclodextrin interfere in the chemical environment around the $\mathrm{Eu}^{3+}$ ions as a second coordination sphere. The changes observed in the band profiles and in the relative intensities of the Stark components of the ${ }^{5} \mathrm{D}_{0} \rightarrow{ }^{7} \mathrm{~F}_{J}$ transitions, at 298 and $77 \mathrm{~K}$, showed that the cyclodextrin acts selectively on the ligand field and $4 \mathrm{f}-4 \mathrm{f}$ intensities, with respect to the different site symmetries.

From the luminescence spectra of the $\mathrm{Eu}^{3+}$ ions in the precursor salt and in the inclusion compound the intensity parameters were obtained. The high values of the $\Omega_{2}$ intensity parameter are in agreement with the hypersensitive character of the ${ }^{5} \mathrm{D}_{0} \rightarrow{ }^{7} \mathrm{~F}_{2}$ and indicates that the $\mathrm{Eu}^{3+}$ ion is in a highly polarizable chemical environment. The differences observed in the intensity parameters for these compounds indicate that steric effects are more significant in the supramolecular species.

\section{Acknowledgements}

The authors acknowledge the financial support from FAPESP, CNPq, FAPEMIG (Brazilian agencies). 


\section{References}

[1] J. Szejtli, Cyclodextrin Technology, Kluwer, Dordrecht, 1989.

[2] W. Saenger, Angew Chem. Int. Ed. Engl. 19 (1980) 344.

[3] M.L. Bender, M. Komiyana, Cyclodextrin Chemistry, Springer, New York, 1977.

[4] R.D. Sinisterra, R. Najjar, O.L. Alves, P.S. Santos, C.A Alves de Carvalho, A.L. Conde da Silva, J. Include. Phenom. 22 (1995) 91.

[5] J.-C.G. Bünzli, G.R. Choppin (Eds.), Lanthanide Probes in Life, Chemical and Earth Sciences-Theory and Practice, Elsevier, Amsterdam, 1989.

[6] G. Blasse, B.C. Grabmaier, Luminescence Materials, Springer, Heidelberg, 1994.

[7] O.L. Malta, Mol. Phys. 42 (1981) 65.

[8] A. Perrotto, R.G. Charles, J. Inorg. Nucl. Chem. 26 (1964) 373.
[9] J.L. Ford, P. Timmins, Pharmaceutical Thermal Analysis-Techniques and Applications, Ellis Horwood, Chichester, UK, 1989.

[10] O.L. Alves, S.F. Fonseca, J. Incl. Phenom. 7 (1989) 589.

[11] C. Brecher, H. Samelson, A Lempicki, J. Chem. Phys. 43 (3) (1965) 1081.

[12] O.L. Malta, H.F. Brito, J.F.S. Menezes, F.R. Gonçalves e Silva, S. Alves Jr., F.S. Farias Jr., A.V.M. de Andrade, J. Lumin. 75 (1997) 255.

[13] O.L. Malta, M.A. Couto dos Santos, L.C. Thompson, N.K. Ito, J. Lumin 69 (1996) 77.

[14] W.T. Carnall, H. Crosswhite, H.M. Crosswhite, Energy Structure and Transition Probabilities of the Trivalent Lanthanides in $\mathrm{LaF}_{3}$, Argonne National Laboratory Report, unnumbered, 1977.

[15] H.F. Brito, O.L. Malta, C.A.A. Carvalho, J.F.S. Menezes, L.R. Souza, R. Ferraz, J. Alloys Comp. 275277 (1998) 254. 